\title{
MS35-P14 | CRYSTAL StRUCtURE AND SELF-ASSEMbLY OF PILLAR[N]PYRIDINIUMS
}

Danylyuk, Oksana (Institute of Physical Chemistry Polish Academy of Sciences, Warsaw, POL); Rosa, Bartlomiej (Institute of Organic Chemistry Polish Academy of Sciences, Warsaw, POL); Butkiewicz, Helena (Institute of Physical Chemistry Polish Academy of Sciences, Warsaw, POL); Kosiorek, Sandra (Institute of Physical Chemistry Polish Academy of Sciences, Warsaw, POL); Boinski, Tomasz (Institute of Organic Chemistry Polish Academy of Sciences, Warsaw, POL); Szumna, Agnieszka (Institute of Organic Chemistry Polish Academy of Sciences, Warsaw, POL); Sashuk, Volodymyr (Institute of Physical Chemistry Polish Academy of Sciences, Warsaw, POL)

Macrocyclic host molecules are important multidentate toolboxes in modern supramolecular chemistry. Depending on their size, shape and electronic properties macrocycles found many useful applications in diverse host-guest transport systems, sensing, extraction, catalysis etc. Very recently, the new class of cationic macrocycles, pillar[n]pyridiniums $\mathrm{P}[n] \mathrm{P}$, has been synthesized and introduced into supramolecular arena. [1] Pillar[n]pyridiniums are cyclic oligomers consisted of pyridynium units linked through nitrogen and para carbon with methylene bridges. The open cylindrical cavity surrounded by cationic electron-deficient pyridinium units provide a highly potential platform for anion recognition and anion-induced self-assembly. Moreover, the simple structure, straightforward synthesis and good aqueous solubility are attractive advantages of these novel macrocyclic hosts.

We would like to present structural aspects and solid state self-assembly features of these inherently cationic macrocycles. Particularly, the conformational properties in the solid state (rigidity versus flexibility of the macrocyclic skeleton), the binding of different anionic guests, main non-covalent interactions involved in the molecular recognition and self-assembly processes with anions will be discussed.

[1] S. Kosiorek, B. Rosa, T. Boinski, H. Butkiewicz, M .P. Szymanski, O. Danylyuk, A.Szumna, V. Sashuk, Chem Commun., 53 (2017) 13320; S. Kosiorek, H. Butkiewicz, O. Danylyuk, V. Sashuk, Chem Commun., 54 (2018), 6316. 